\title{
Hypofractionated radiotherapy for lung tumors with online cone beam CT guidance and active breathing control
}

\author{
Yali Shen ${ }^{1}$, Hong Zhang ${ }^{1}$, Jin Wang ${ }^{1}$, Renming Zhong ${ }^{2}$, Xiaoqing Jiang ${ }^{2}$, Qinfeng $X u^{2}$, Xin Wang ${ }^{1}$, Sen Bai ${ }^{*}$, \\ Feng $\mathrm{Xu}^{1 *}$
}

\begin{abstract}
Background: To study the set-up errors, PTV margin and toxicity of cone beam CT (CBCT) guided hypofractionated radiotherapy with active breathing control $(A B C)$ for patients with non-small cell lung cancer (NSCLC) or metastatic tumors in lung.
\end{abstract}

Methods: 32 tumors in 20 patients were treated. Based on the location of tumor, dose per fraction given to tumor was divided into three groups: 12 Gy, 8 Gy and 6 Gy. ABC is applied for every patient. During each treatment, patients receive $\mathrm{CBCT}$ scan for online set-up correction. The pre- and post-correction setup errors between fractions, the interfractional and intrafractional, set-up errors, PTV margin as well as toxicity are analyzed.

Results: The pre-correction systematic and random errors in the left-right (LR), superior-inferior (SI), anteriorposterior (AP) directions were $3.7 \mathrm{~mm}$ and $5.3 \mathrm{~mm}, 3.1 \mathrm{~mm}$ and $2.1 \mathrm{~mm}, 3.7 \mathrm{~mm}$ and $2.8 \mathrm{~mm}$, respectively, while the post-correction residual errors were $0.6 \mathrm{~mm}$ and $0.8 \mathrm{~mm}, 0.8 \mathrm{~mm}$ and $0.8 \mathrm{~mm}, 1.2 \mathrm{~mm}$ and $1.3 \mathrm{~mm}$, respectively. There was an obvious intrafractional shift of tumor position. The pre-correction PTV margin was 9.5 $\mathrm{mm}$ in LR, $14.1 \mathrm{~mm}$ in SI and $8.2 \mathrm{~mm}$ in AP direction. After CBCT guided online correction, the PTV margin was markedly reduced in all three directions. The post-correction margins ranged 1.5 to $2.1 \mathrm{~mm}$. The treatment was well tolerated by patients, of whom there were 4 (20\%) grade1-2 acute pneumonitis, 3 (15\%) grade1 acute esophagitis, 2 (10\%) grade1 late pneumonitis and 1 (5\%) grade 1 late esophagitis.

Conclusion: The positioning errors for lung SBRT using ABC were significant. Online correction with CBCT image guidance should be applied to reduce setup errors and PTV margin, which may reduce radiotherapy toxicity of tissues when $A B C$ was used.

\section{Background}

Radiotherapy is the alternative treatment for patients with medically inoperable primary non-small-cell lung cancer (NSCLC) [1], and also for patients with slow growing metastatic lung tumors [2] which when managed with high dose localized radiotherapy can prolong patients' symptom-free status.

However, even for inoperable stage I non-small cell lung cancer, the local control rates using standard fractionation schemes (30-76 Gy in 1.8 to 2.0 Gy fractions)

\footnotetext{
* Correspondence: tonybaisen@yahoo.com.cn; fengxuster@gmail.com

'Department of radiation oncology, Cancer centre, West China Hospital, Sichuan University, Chengdu 610041, China

${ }^{2}$ Division of Physics Center, Cancer centre, West China Hospital, Sichuan 2Division of Physics Center, Cancer
University, Chengdu 610041, China
}

have been reported ranging 45-89\% [3-5]. Five year actuarial survival of conventional radiotherapy ranged from $6 \%$ to $27 \%$ [6-9], which was unsatisfactory compared with surgery (with a 5 -year survival rate of $60 \%$ to 80\%) [10]. Dose escalation has been an important issue to improve local tumor control and overall survival $[11,12]$. However, dose escalation by conventional fractionated radiotherapy has the risk of increasing normal tissue toxicity and prolonging overall treatment time which will encounter the acceleration of tumor cell proliferation.

The dose escalation within a short treatment time and sparing functional lung tissue is potentially addressed by hypofractionated radiotherapy. It has been shown that 
the use of hypofractionated lung radiotherapy can achieve excellent local control rates as high as $85-95 \%$, with surprisingly minimal acute or late toxicity [13-15]. The hypofractionation radiotherapy technique employs multiple radiation beams to target a tumor with extreme precision, delivering a high dose of radiation, even in a single fraction. Tumors in the thorax regions are subject to setup errors and respiration motion, which can result in inaccurate assessment of organ shape and locations. Conventionally, these uncertainties are accounted for in treatment planning by using large margins based on motion value [16], which can limit dose delivered to tumor.

Special immobilization and verification devices have been developed to reduce setup uncertainties. The use of cone-beam CT (CBCT) has provided 3-dimension information of patient position which could be utilized to guide high precision radiotherapy of the lung tumor. The technique of active breathing control (ABC) has been used to reduce the breathing motion. The use of $\mathrm{ABC}$ has been reported to have advantages in protection of lung tissues by reducing respiration motion and lung density [17]. However, little has been reported on the combined use of $\mathrm{ABC}$ and $\mathrm{CBCT}$ in hypofractionated $\mathrm{RT}$ of lung tumor.

Given the availability of onboard cone-beam CT (CBCT) imaging and $\mathrm{ABC}$ at our institution, we set out to determine how much using image-guided radiotherapy (IGRT) might affect lung tumor targeting accuracy, target volume margin requirements, and normal tissue doses.

\section{Methods}

\section{Study population and Characterization} Eligibility

Patients with histologically or cytologically confirmed diagnosis of metastatic malignant tumors within the lung or primary NSCLC were eligible for treatment. Patients must have measurable disease and the maximum diameter of tumors is bellow $5 \mathrm{~cm}$. A maximum of 3 lung tumor targets in one patient were allowable. Patients with primary NSCLC either had medically inoperable disease or refused surgery. Patients with metastatic tumors and with life expectancy $\geq 6$ months were treated. Patients with a history of prior chest radiotherapy were ineligible. Pretreatment pulmonary function testing was performed, with FEV1 (minimum forced expiration volume at 1 second) $\geq 2.0 \mathrm{~L}$ and FEV1/FVC (vital capacity) $\geq 80 \%$. Patients were required to have an ECOG performance status of 2 or less, and not on chemotherapy or hormonal therapy. Informed consent was obtained from all patients before the treatment was initiated.

Patient characteristics are shown in Table 1

Between April 2006 and August 2007, 20 patients with inoperable NSCLC or metastatic lung tumors were treated with IGRT at West China Hospital, Sichuan University. Patients comprised 12 males and 8 females aged from 22 to 74 years, with a median age of 54.2 years. Half of the lung tumors were primary NSCLC and the rests were metastatic which came from the head and neck (7), esophagus (2) and breast (1). Of the 10 patients with primary NSCLC, 5 were recurrence after surgical treatment, 3 were ineligible for surgical treatment due to complications and/or advanced age and 2 refused surgery. Ten patients had 1 tumor target, eight patients had 2 targets and the remaining two patients had 3 targets. A total of 32 tumor targets were treated with radiotherapy. The tumor mean size was $23 \mathrm{~mm}$ (ranged $13-44 \mathrm{~mm}$ ) on CT scan.

\section{Immobilization and CT simulation}

All patients underwent a virtual radiation simulation using a stereotactic body frame (SBF) (Elekta Crawley, UK) for immobilization. A planning CT scan in $3 \mathrm{~mm}-$ cuts of the whole thorax was taken, with the patient in the treatment position and using the Elekta $A B C$ device (Elekta, Crawley, UK). To set the threshold of ABC, the patient was told to take a deep breath and the maximum inspiration volume was measured. The breathhold threshold was set at $3 / 4$ of the maximum

\section{Table 1 Patient and tumor characteristics}

\begin{tabular}{ll}
\hline Patient & 20 \\
Tumor & 32 \\
Age (yrs) & \\
Range & $22-74$ \\
Median & 54.2 \\
$\geq 60$ yrs & $8(40 \%)$ \\
Gender & \\
Male 19 & $12(60 \%)$ \\
Female & $8(40 \%)$ \\
Resource & \\
Primary & 10 \\
Metastatic & 10 \\
Histology & \\
Squamous cell carcinoma & $5(25 \%)$ \\
Adenocarcinoma & $11(55 \%)$ \\
Unclassified & $4(20 \%)$ \\
Tumor size (mm) & \\
Mean & 23 \\
Range & $13-40$ \\
Tumor location & \\
Right lung & $20(63 \%)$ \\
Left lung & $12(37 \%)$ \\
Upper lobe & $12(37 \%)$ \\
ECOG performance & \\
0 & $14(70 \%)$ \\
1 & $6(30 \%)$ \\
\hline
\end{tabular}


inspiration for each patient. Each patient had accepted the training course with $\mathrm{ABC}$ for 2-5 times before irradiation. Oxygen with 5-8 L flow rate was connected to the inhale pipe to help patients enhance breath holding time. Patients can release the control switch when he feels uncomfortable. It is required that the respiration motion of tumor with $A B C$ should be $<3 \mathrm{~mm}$ as assessed by fluoroscopy before treatment.

\section{Planning and treatment}

Treatment planning was performed using the PrecisePLAN Release 2.1 planning system with considerations made for pulmonary density inhomogeneity. The full area integration dose calculation algorithm was used for dose calculation. Prophylactic nodal irradiation was not performed. Gross tumor volume (GTV) encompassed 1 $\mathrm{mm}$ only the radiologically visible tumor as seen by the planning $\mathrm{CT}$ with the lung window using a window level of -700 with a width of 1000 . Clinical target volume (CTV) was GTV plus a $5 \mathrm{~mm}$ margin in all directions. For the planning target volume (PTV), $5 \mathrm{~mm}$ security margins in all directions were added to the CTV.

Depending on tumor size and location, different fractionation schemes were applied. There were three groups of different dose per fraction given to the planning target volume (PTV), prescribed to the $80 \%$ isodose. In general, radiotherapy with dose per fraction of 12 Gy prescribed was chosen for small targets and for targets with peripheral location. In cases of large tumors, central location and close proximity to critical structures like large vessels and bronchi: with dose per fraction of $6 \mathrm{~Gy}$. Other tumors were given radiotherapy with dose per fraction of $8 \mathrm{~Gy}$. Depend on different single dose we chose different numbers of fractions to make BED (biology effective dose) reach at least 70 Gy. The primary and metastatic lung tumors were not differentially fractionated since in this cohort both primary and metastatic tumors shared similar histopathological types with similar radiosensitivity. The patients received radiotherapy three times per week. The treatment planning ensured that the esophagus, heart and spinal cord received the minimum possible dose, but always less than $50 \%$ of the total prescribed tumor dose. According to linear quadratic equation $[E / \alpha=n d \times[1+d /(\sigma / \beta)]$, BED were calculated and shown in Table 2 .

\section{$\mathrm{CBCT}$ guidance and adjustment}

CBCT was used for verification of tumor position using100 kV, S20 field of view $(270 \mathrm{~mm}), 36.1 \mathrm{mAs}$, with the kilovoltage source rotating from $260^{\circ}$ and ending at $100^{\circ}$ for acquisition of 361 frames [18], which was done in one breath-hold.

Before each fraction, a first $\mathrm{CBCT}$ was acquired reconstructed and automatically matched to the planning CT.
Table 2 Radiation therapy fractionations protocols according to Abratt model.

\begin{tabular}{cccc}
\hline $\begin{array}{c}\text { Targets } \\
\text { numbers }\end{array}$ & $\begin{array}{c}\text { Dose (Gy) } \\
\text { infraction }\end{array}$ & $\begin{array}{c}\text { Number of } \\
\text { fractions }\end{array}$ & $\begin{array}{c}\text { BED } \\
\text { (Gy) }\end{array}$ \\
\hline 18 & 12 & 4 & 106 \\
5 & 8 & 7 & 101 \\
3 & 8 & 5 & 72 \\
3 & 6 & 10 & 96 \\
3 & 6 & 8 & 77 \\
\hline
\end{tabular}

* BED: biological effective dose.

The positional errors of the target in left-right (LR), superior-inferior (SI) and anterior-posterior (AP) axis were calculated with the XVI software. The errors were corrected online through adjustment of treatment couch. The second CBCT was acquired after online correction. If the residual error is less than $2 \mathrm{~mm}$, radiotherapy was delivered immediately. The third CBCT were acquired after radiotherapy to estimate the residual error. The interfractional errors were defined as the offset between the pre-correction $\mathrm{CBCT}$ and the planning $\mathrm{CT}$. The intrafraction error was calculated as the difference between the pre- and post-correction position.

\section{Analysis of positional errors}

The inter- and intra-fraction errors are reported as described [19]: for each patient the mean and standard deviation (SD) of all setup errors during treatment were calculated. The group mean error $(M)$ is defined as the average of all individual means. $\Sigma$ is defined as the variability of the means and calculated as the SD of the individual means. The random uncertainty $\sigma$ was calculated as the root-mean-square of the individual SD.

\section{PTV margin reduction and impact on normal tissue dose}

As $\mathrm{ABC}$ was applied to restrict respiration motion $(<3 \mathrm{~mm})$, to simplify analysis, the internal margin (IM) due to respiration motion was not included for margin analysis, only positional uncertainty was accounted for, according to van Herk [19]: $\mathrm{M}_{\text {setup }}$ (PTV margin) $=2.5 \Sigma+$ $\beta \sqrt{ } \sigma^{2}+\sigma_{p}^{2}-\beta \sigma_{p}$, where $\sigma_{p}=6.4 \mathrm{~mm}$ for lung, $\beta=0.84$ for SBRT (80\% isodose line) [20].

To evaluate the benefit of CBCT-guided online setup correction and $A B C$ device on margin reduction for lung hypofractionated RT, the dose reductions to normal tissues with online correction were simulated in three patients with central, peripheral, and inferior lobe tumor locations, respectively.

\section{Follow up}

Acute toxicity was prospectively assessed for lung, esophagus, and skin using the RTOG acute radiation morbidity scoring criteria every week during treatment. Late 
lung toxicity was evaluated with a modified scoring system considering only the lung symptoms (Common Toxicity Criteria version 2). Our follow up lasts 16 month. The patients got recheck for chest CT every 3 months. The tumor response was evaluated by a senior radiologist and a radiation oncologist using the RECIST criteria.

\section{Statistical analysis}

F-test was applied for error analysis using SPSS software package.

\section{Results}

A total of 347 CBCT including 150 pre-correction, 130 post-correction and 67 post-treatment scans were acquired. The $\mathrm{CBCT}$ images with $\mathrm{ABC}$ yielded good contrast of tumor and structures.

At free breathing, the mean $( \pm \mathrm{SD})$ of diaphragm displacement was $16.0( \pm 2.7) \mathrm{mm}$ (range $12-24 \mathrm{~mm})$. With the use of $\mathrm{ABC}$, the mean $( \pm \mathrm{SD})$ diaphragm displacement was $1.7( \pm 0.5) \mathrm{mm}$ (range 1.0-2.5 mm). Mean value of the breath-hold time was prolonged from 30 seconds to 57 seconds by means of training course and inhaling oxygen in 20 patients. All patients tolerated $A B C$ well for $C B C T$ scan with a duration $\geq 40 \mathrm{~s}$. To make patients more comfortable, the duration of $\mathrm{ABC}<$ $20 \mathrm{~s}$ is required during radiation delivery. All patients were tested by the respiratory function 3 months after treatment. The result indicated that none of these parameters (FEV1, FEV1/FVC) was affected by IGRT.

\section{Interfractional errors and intrafracional errors}

The pre- and post-correction positional errors for patients using $\mathrm{ABC}$ in LR, SI and AP directions were shown in Fig. 1 and Fig. 2. It shows that errors in three directions were all decreased with online correction. The percentage of pre-correction errors $\leq 2 \mathrm{~mm}$ in all three directions was $<30 \%$, while rose to $\geq 90 \%$ after correction and remained $60 \%$ at the completion of treatment. As shown in Table 3, the pre-correction errors in SI direction were the largest. There was a significant deviation of mean error in the caudal direction, with systematic error of $5.3 \mathrm{~mm}$ and random error of $3.7 \mathrm{~mm}$. The precorrection errors were similar in LR and AP directions, with the systematic of 3.1 and 3.7 $\mathrm{mm}$ and random error of 2.1 and $2.8 \mathrm{~mm}$ for LR and AP, respectively. After correction, the errors were reduced in all three axes. The errors were similar in three axes, with the systematic errors of $0.6 \mathrm{~mm}$ in LR and $0.8 \mathrm{~mm}$ for both SI and AP direction; and random errors of $0.8 \mathrm{~mm}, 1.2 \mathrm{~mm}$ and $1.3 \mathrm{~mm}$ in LR, SI and AP direction, respectively.

After treatment, the tumor positional errors increased compared to post-correction (Table 3). The systematic errors were $1.2 \mathrm{~mm}$ for both LR and AP axes, and larger $(1.8 \mathrm{~mm})$ in SI direction. The random error was larger in AP $(2.8 \mathrm{~mm})$ than in LR and SI (both1.5 $\mathrm{mm}$ ) direction. The mean errors were all below $0.5 \mathrm{~mm}$. The post-treatment residual errors were larger than the postcorrection, with increments $\leq 1 \mathrm{~mm}$, but still much smaller than pre-correction errors.

\section{PTV margin reduction and impact on normal tissue dose}

In this study, with $A B C$ device, the respiration motion of tumor was small, only setup uncertainty was included for margin analysis. In Table 3, the pre-correction setup margin was largest $(14.1 \mathrm{~mm})$ in SI, intermediate in LR $(9.5 \mathrm{~mm})$ and smallest in AP $(8.2 \mathrm{~mm})$ directions. The margins decreased markedly after correction to within 3 $\mathrm{mm}$ in all directions. After treatment the margins increased to $3.2-4.7 \mathrm{~mm}$ in three directions.

Table 4 shows the reductions in normal tissue dose volume parameters using online $\mathrm{CBCT}$ image guidance for each of the three GTV locations (central, peripheral, and lower lobe). Comparisons are made between different PTV scenarios. In patients using $\mathrm{ABC}$, the reductions of $47-77.3 \%$ in lung dose volume endpoints were achieved with $\mathrm{CBCT}$ correction. Reductions of $36.3-66.7 \%$ in lung dose volume endpoints were achieved when intrafractional setup errors were accounted for. In patients using $A B C$, reduction in spinal cord doses was highest (55.2-58.5\%) for central tumor location and smaller (8.4-17\%) for peripheral locations. Comparing to precorrection (PTV1), the dose reductions to normal tissues were greater postcorrection (PTV2) than posttreatment (PTV3). The increment of dose reductions in lung dose volume endpoints ranged 9.1-18.8\%, and 3.3-5.9\% in spinal cord maximum dose in PTV2 compared to PTV3, suggesting increased normal tissue dose with posttreatment margin due to intrafractional positioning errors.

\section{Follow up}

ll patients were followed up for 6 - 16 months, with a median of 10 months. There was one patient dying of brain metastasis. At 6 months post-treatment, 20 $(62.5 \%)$ out of 32 targets regressed completely after treatment. 9 (28\%) targets shrank more than 30\% (PR). $2(6.3 \%)$ targets had SD at 6 months post-treatment, 1 target was not assessed. An overall response rate of 90.6\% (29/32) was achieved. The CR was higher in patients with BED $\geq 100$ Gy (74\%) vs. BED < 100 Gy (33\%). The maximum dose of the critical organs was well below the tolerance dose for each organ in the whole group. The maximum value of V20 for the whole group was $21 \%$. The maximum point dose of spinal cord, esophagus and mean lung were 15.7 Gy, 32 Gy and 3.0 Gy respectively. Treatment was well tolerated. 


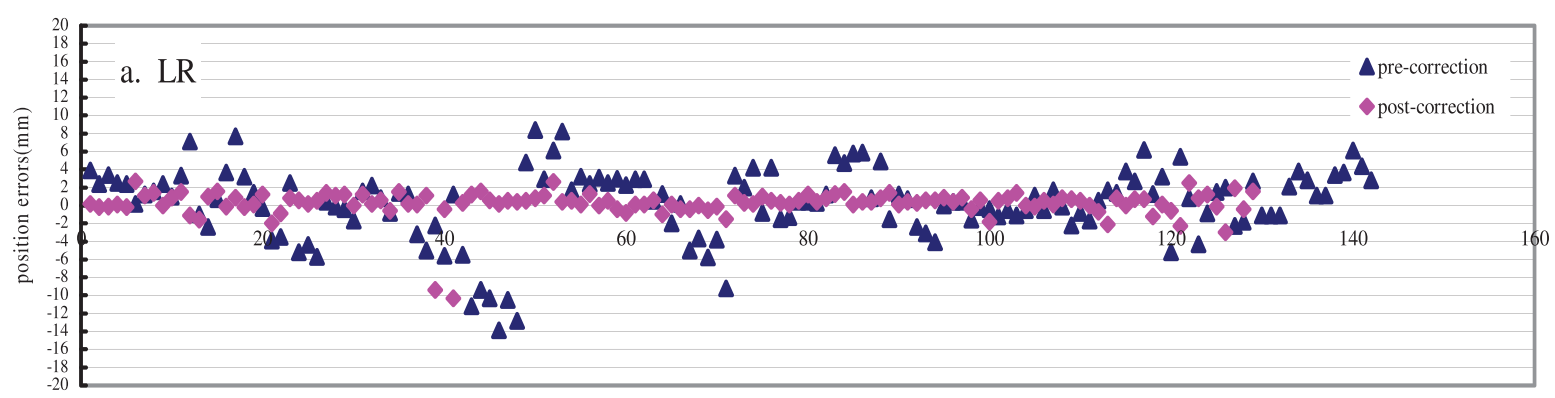

CBCT numbers

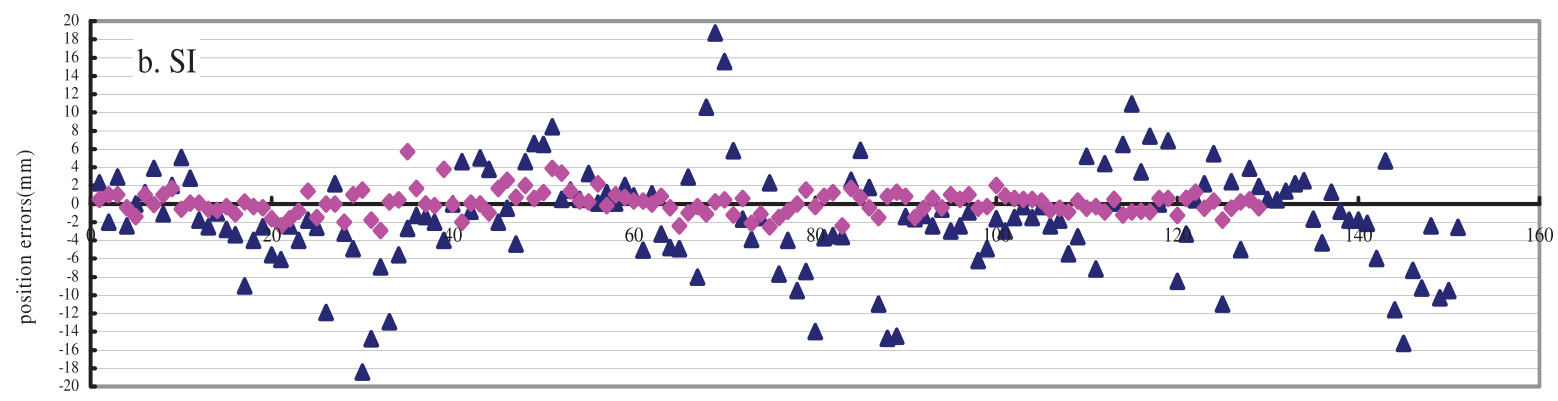

CBCT numbers

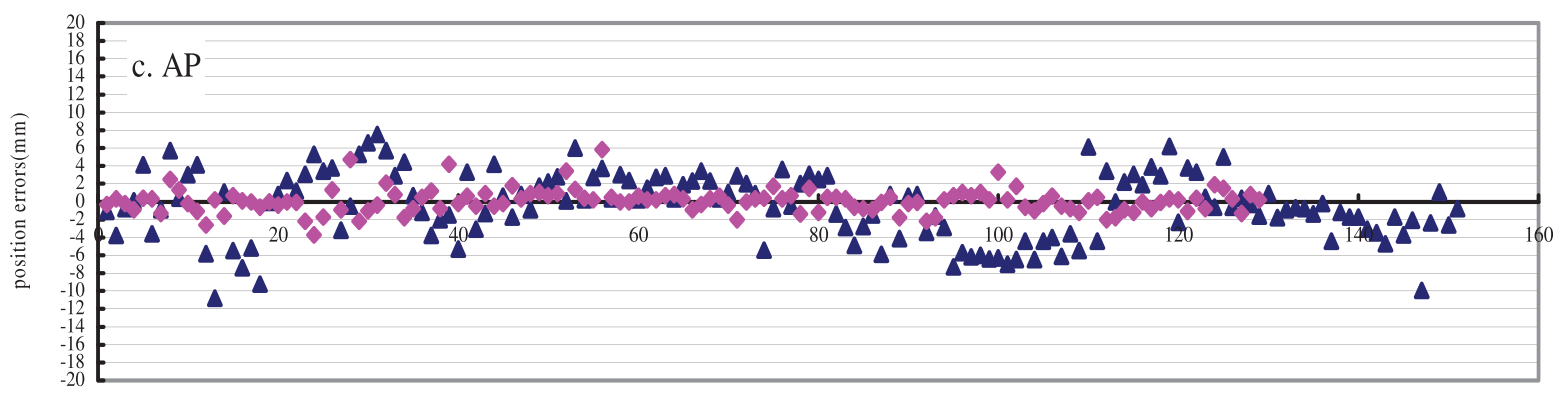

CBCT numbers

Figure 1 The tumor positional errors pre- and post-correction, and post-treatment in three dimensions. The abscissa represents the number of $C B C T$ acquired, and the ordinate represents the errors (in $\mathrm{mm}$ ), a: $L R, b: S I, c: A P$.

Majority of patients did not have treatment related symptoms during and after treatment. $3(15 \%)$ patients had grade $1,1(5 \%)$ patient had grade 2 acute radiation pulmonary toxicity. 3 (15\%) had grade 1 acute esophagitis. All these symptoms alleviated after treatment completion without special treatment. There was no pulmonary or esophageal toxicity of grade 3 or above, no acute skin toxicity and no hemotoxicity during treatment. For late effects, only $2(10 \%)$ patients had grade 1 pulmonary toxicity (imaging change but no symptom), 1 patient (5\%) had grade 1 dysphasia. No other late toxicities were observed.

\section{Discussion}

In this preliminary study, we evaluated the feasibility of $\mathrm{CBCT}$ guided radiotherapy in combination with $\mathrm{ABC}$ to restrict tumor positional error. The role of CBCT guidance in improving treatment accuracy and reduction of target margin requirements for stereotactic lung radiotherapy using $\mathrm{ABC}$ procedure was studied.

At initial setup, the tumor positional error was significant even with SBF plus ABC. Our results were similar to literature report that utilized SBF immobilization and portal imaging device to evaluate errors [21] which reported the positioning errors for SBF were 2.3-4.2 $\mathrm{mm}$. Negoro [22] also reported the positioning accuracy ranged $0-8.5 \mathrm{~mm}$, with the mean of $3.2 \mathrm{~mm}$. Our results demonstrated that the initial errors with SBF plus ABC immobilization were greater than those reported recently which also utilized $\mathrm{CBCT}$ online guidance and 4D-CT to detect errors in lung tumor immobilized with SBF alone. In their study, the systematic error ranged 

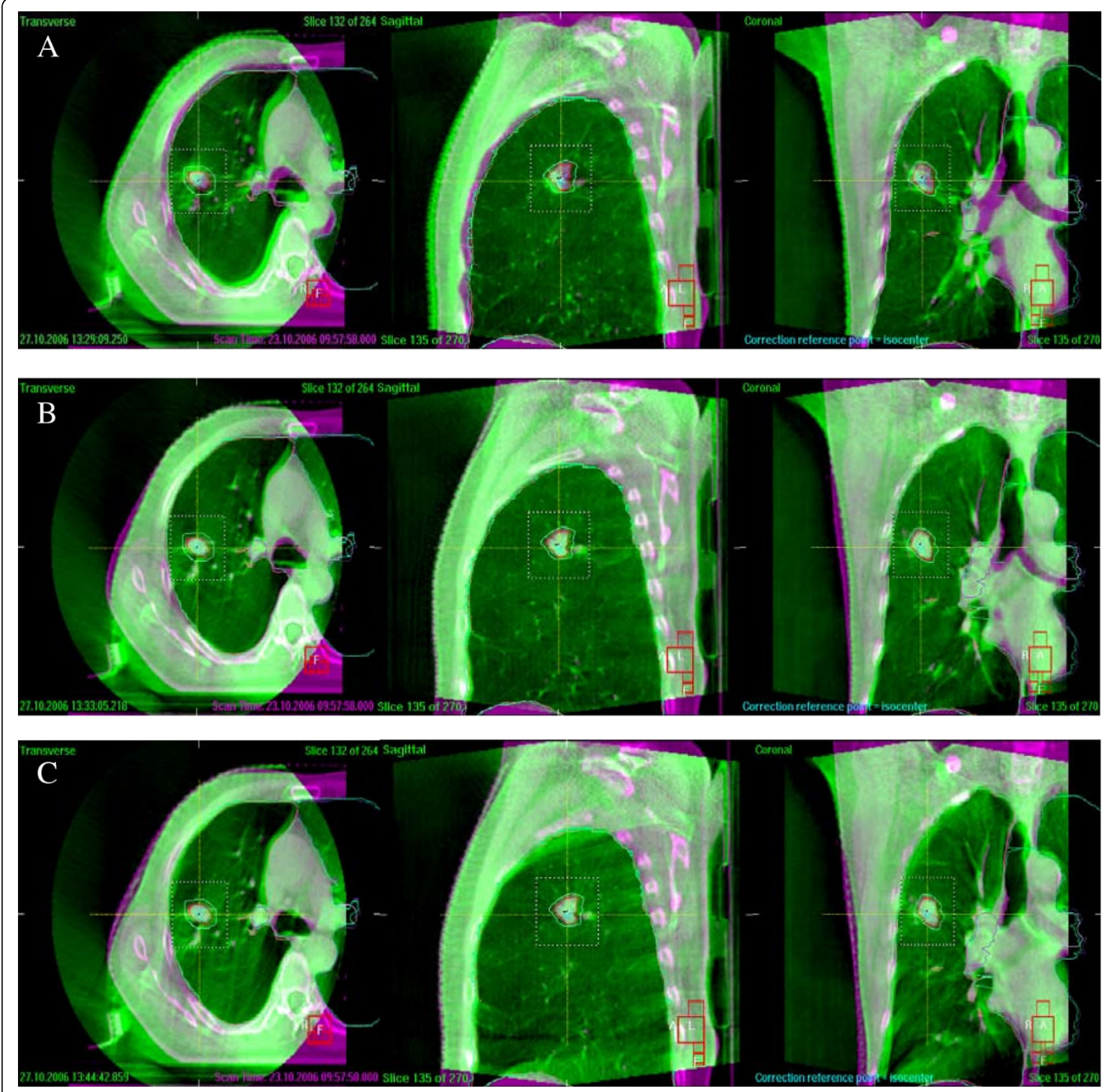

Figure 2 The ovelapping of targets between simulation CT and CBCT scans of a right upper lung NSCLC. The pink circle represents the GTV and green circle represents the PTV contours in planning CT. From left to right: transverse, sagital and coronal. A: precorrection, shows shifts of the target position from planning contours. B: Post-correction with online correction, shows satisfactory overlapping of the contours between simulation and $\mathrm{CBCT}$ images. C: Post-treatment, the targets in $\mathrm{CBCT}$ still overlaps well with the contours in planning $\mathrm{CT}$.

2.5 to $3.4 \mathrm{~mm}$ and random errors ranged 1.7 to $2.7 \mathrm{~mm}$ [16]. This may be partly due to the poor long-term reproducibility of tumor position when repeat CT scans were performed during $A B C$ [23]. It should be mentioned that the value of using the SBF for improving setup accuracy in SBRT is controversial. In a recent study reported by Sonke et al [20], 65 patients with small peripheral lung tumors treated with SBRT without a SBF. In their study the positioning accuracy was evaluated using 4DCT and CBCT imaging, and their results were similar to ours. Although online correction markedly reduced the positional error, the tumor position varied during treatment and might affect the dose distribution in stereotactic radiotherapy. The post-treatment residual systematic errors increased, with the greatest increment of $1 \mathrm{~mm}$ in SI direction, and $0.4 \mathrm{~mm}$ for both LR and AP direction. The increment of intrafractional random error was $1.5 \mathrm{~mm}$ in AP, $0.3 \mathrm{~mm}$ in 
Table 3 The systematic and random errors and PTV margins in $\mathbf{2 0}$ patients pre- and post-correction, and post-treatment.

\begin{tabular}{cccccccccc}
\hline Setup error & \multicolumn{2}{c}{$\begin{array}{c}\text { Pre-correction } \\
(\mathbf{N}=\mathbf{1 5 0})\end{array}$} & \multicolumn{2}{c}{$\begin{array}{c}\text { Post-correction } \\
(\mathbf{N}=\mathbf{1 3 0})\end{array}$} & \multicolumn{2}{c}{$\begin{array}{c}\text { Post-treatment } \\
(\mathbf{N}=(\mathbf{6 7})\end{array}$} \\
\cline { 2 - 10 } & LR & SI & AP & LR & SI & AP & LR & SI & AP \\
\hline$M$ & -0.3 & -1.74 & -0.5 & 0.1 & 0.1 & 0.1 & -0.4 & 0.3 & 0.4 \\
$\Sigma$ & 3.7 & 5.3 & 3.1 & 0.6 & 0.8 & 0.8 & 1.2 & 1.8 & 1.2 \\
$\sigma$ & 2.1 & 3.7 & 2.8 & 0.8 & 1.2 & 1.3 & 1.5 & 1.5 & 2.8 \\
$M_{\text {setup }}$ & 9.5 & 14.1 & 8.2 & 1.5 & 2.1 & 2.1 & 3.2 & 4.7 & 3.5 \\
\hline
\end{tabular}

Abbreviations: $\mathrm{N}=$ number of $\mathrm{CBCT}$ scans; $\mathrm{M}=$ group mean error; $\Sigma=\mathrm{SD}$ of individual means for setup error; $\sigma=$ root-mean-square of individual $S D$ for setup error; $\mathrm{LR}=$ left-to-right; $\mathrm{SI}=$ superior-inferior; $\mathrm{AP}=$ anterior-posterior; $M_{\text {setup }}=$ PTV margin for setup. $M_{\text {setup }}($ PTV margin $)=2.5 \Sigma+\beta \sqrt{ } \sigma^{2}+\sigma_{p}^{2}-\beta \sigma_{p}$

SI and $0.8 \mathrm{~mm}$ in LR direction. Only few studies have reported on intrafractional tumor position variation, especially in patients applying ABC. Uematsu et al. [24] used CT scan to measure the intrafraction lung tumor position error and observed that the intrafraction positional variation was small. Guckenberger et al. [25] has utilized $\mathrm{CBCT}$ to determine intrafractional error and postulated that $90 \%$ of the intrafractional position errors were within $4.8 \mathrm{~mm}$. A recent literature [18] reported that the mean (SD) intrafractional errors of $-0.1 \mathrm{~mm}$ $(1.1 \mathrm{~mm}), 0.2 \mathrm{~mm}(1.4 \mathrm{~mm})$ and $-0.1 \mathrm{~mm}(1.5 \mathrm{~mm})$ in LR, SI and AP axes respectively, for thoracic tumors at free breathing, which were smaller than this cohort of patients. This implies that using of $\mathrm{ABC}$ might increase the intrafractional patient motion. This might be due to the using of $\mathrm{ABC}$ which introduces more procedures and variations [16].

Based on our study, the use of $A B C$ has reduced respiration motion. With $\mathrm{ABC}$, the average diaphragm displacement was significantly reduced from $16.0 \mathrm{~mm}$ to $1.7 \mathrm{~mm}$. Hanley et al. [26] has reported that the diaphragm motion was reduced from $26.4 \mathrm{~mm}$ to $2.5 \mathrm{~mm}$ (0.5-4.9 mm) with ABC procedure. Sarrut [27] reported the lung tumor motion of 0.9-5.9 $\mathrm{mm}$ with the use of ABC. Our study also showed high reproducibility of 2 $A B C$ procedures, with a diaphragm movement error of $3 \mathrm{~mm}$. However, there is limitation of using diaphragm position as a surrogate for tumor. This is because that the diaphragm is susceptible to imaging artifacts due to large and rapid motion, and the diaphragm motion may also be influenced by nonrespiratory activity [28].

As ABC was applied to all patients in this study, the tumor respiration motion was small $(<3 \mathrm{~mm})$, the internal target volume (ITV) was considered roughly equal to CTV. Compared to the reliability of tumor motion (average displacement being $1.4 \pm 1.0 \mathrm{~mm}$ ) measured by $4 \mathrm{D}$ CT [29], the reliability of tumor motion measured by fluoroscopy is similar. For simplification, the PTV margin calculation only considered setup errors, other error sources such as delineation uncertainty and breathing pattern variation were not accounted for in this study. The pre-correction PTV margin was $9.5 \mathrm{~mm}$ in LR, 14.1 $\mathrm{mm}$ in SI and $8.2 \mathrm{~mm}$ in AP direction. However, it was recommended a uniform PTV margin of $5 \mathrm{~mm}$ axial and $10 \mathrm{~mm}$ superior-inferior be added for stereotactic lung radiotherapy when image guidance is not used [16]. Our results showed that the margins in three axes all exceeded the recommended margins for a magnitude about $5 \mathrm{~mm}$ if CBCT guidance not applied, indicating the necessity of image guidance for accuracy of lung SBRT with ABC. After CBCT guided online correction, the PTV margin was markedly reduced in all three directions. The post-correction margins ranged 1.5 to $2.1 \mathrm{~mm}$

Table 4 Reductions in normal tissue dose volume parameters using online CBCT image guidance and ABC for SBRT of three GTV (16 cc) location

\begin{tabular}{|c|c|c|c|c|c|c|}
\hline \multirow[t]{2}{*}{ Normal tissue parameter } & \multicolumn{3}{|c|}{ Dose absolute reduction value } & \multicolumn{3}{|c|}{ Dose reduction $\%$} \\
\hline & PTV1 & PTV2 & PTV3 & $\begin{array}{l}\text { from PTV1 } \\
\text { to PTV2 }\end{array}$ & $\begin{array}{l}\text { from PTV1 } \\
\text { to PTV3 }\end{array}$ & from PTV2 to PTV3 \\
\hline \multicolumn{7}{|l|}{ Mean lung dose(Gy) } \\
\hline central location & 5.42 & 2.42 & 2.91 & 55.4 & 46.3 & 9.1 \\
\hline peripheral location & 5.05 & 2.47 & 3.22 & 51.1 & 36.3 & 14.8 \\
\hline inferior lobe of lung & 11.46 & 6.07 & 7.47 & 47.0 & 34.8 & 12.2 \\
\hline \multicolumn{7}{|l|}{ Lung V20 (\%) } \\
\hline central location & 11 & 2.5 & 3.7 & 77.3 & 66.7 & 10.6 \\
\hline peripheral location & 8 & 2.5 & 4 & 68.8 & 50.0 & 18.8 \\
\hline inferior lobe of lung & 21 & 10 & 12 & 52.4 & 42.9 & 9.5 \\
\hline \multicolumn{7}{|c|}{ Spinal cord Maximum dose(Gy) } \\
\hline central location & 59.34 & 24.64 & 26.58 & 58.5 & 55.2 & 3.3 \\
\hline peripheral location & 6.91 & 5.99 & 6.32 & 13.3 & 8.4 & 4.9 \\
\hline inferior lobe of lung & 22.50 & 18.67 & 20.00 & 17.0 & 11.1 & 5.9 \\
\hline
\end{tabular}

Abbreviations: PTV1 = precorrection margins with $A B C$; PTV2 = postcorrection margins with $A B C$; PTV3 = posttreatment residual margin with $A B C+C B C T-$ correction; $\mathrm{V} 20$ = volume of both lungs receiving $\geq 20 \mathrm{~Gy}$. 
which were similar to the literature which utilized CBCT online correction for lung patients [16]. The PTV margins at the completion of treatment were increased as compared to the post-correction margins. The post-treatment margins were $3.2-4.7 \mathrm{~mm}$ in three directions. It was suggested by some investigators that at least $5 \mathrm{~mm}$ margin should be added for individualized PTV if image guidance and SBF is used [30]. When intra-and interfractional errors were both accounted for, the PTV margin reduction with online correction ranged about 5 to 10 $\mathrm{mm}$ in different axes. In our study the online correction resulted in reduction of lung dose volume endpoints of $47-77.3 \%$, and $55.2-58.5 \%$ in spinal cord doses for patients using $\mathrm{ABC}$ at different tumor locations. It could be inferred from our study that $\mathrm{CBCT}$ and online correction can significantly reduce normal tissue doses. As in our study the tumor respiration motion was not evaluated, the benefit of $A B C$ on ITV reduction could not be discussed. It has been indicated that most lung tumors do not exhibit significant motion [31] and there remains inter- breath hold variability in peripheral lung tumor position with the use of $A B C$ inspiration breath hold, which prevents significant PTV margin reduction. However, lung volumes can significantly increase, thereby decreasing the mass of lung within a standard PTV [32]. In addition, $\mathrm{ABC}$ may result in a mean relative reduction in lung DVH parameters determining risk of pneumonitis by up to $25 \%$ with the potential for safe dose escalation as reported in other study [33]. 4DCT scan has become more popular for SBRT, it has been reported that using mid-ventilation CT scans for treatment planning instead of the conventional free-breathing CT scans, margin reduction is possible, which can reduce the treatment volume up to $50 \%$ [34].

Our study found that hypofractionated radiotherapy with BED ranged 72 to 100 Gy could achieve high CR $(62.5 \%)$, The hypofractionated radiotherapy has radiobiological advantages of counteracting tumor accelerated proliferation. Quite a few researches have been published which showed high local tumor control and surprisingly low toxicities with BED of $100 \mathrm{~Gy}$ [35-39]. The response rate in our study seems higher than that reported for NSCLC SBRT, which were assumed to be partly due to the heterogeneity of histopathology in this cohort, since half the cases were metastatic tumors from head and neck, esophagus or breast which were radioresponsive. The small tumor size and small number of cases included in this cohort might be the other contributing factors.

The patients tolerated the treatment well. Only grade 1-2 acute toxicity occurred in $35 \%$ of the patients and $15 \%$ had grade I late toxicity. Though immobilization device combined with $A B C$ could effectively reduce respiration motion of target, the total margin was not reduced. With the use of online CBCT guided setup correction, PTV margin was substantially reduced, which explained the low toxicity in this patient cohort. Similar results were reported by Fukumoto et al [40] who treated 22 stage I patients with image guided hypofractionated radiotherapy (48-60 Gy in 8 fractions) and found nearly no impairment of pulmonary functions.

In conclusion, for lung cancer hypofractionated radiotherapy using $\mathrm{ABC}, \mathrm{CBCT}$ guided online correction effectively reduced setup errors and PTV margins. $\mathrm{CBCT}$ guidance markedly improved the precision of lung SBRT which might offer a potential dose escalation and effective reduction of normal tissue toxicity.

\section{Declaration of interests}

The authors declare that they have no competing interests.

\section{Author details}

'Department of radiation oncology, Cancer centre, West China Hospital, Sichuan University, Chengdu 610041, China. '2Division of Physics Center, Cancer centre, West China Hospital, Sichuan University, Chengdu 610041, China.

\section{Authors' contributions}

YS carries out the design of the study and drafting the manuscript; $\mathrm{HZ}$ and JW worked on analysis of data; RZ, XJ, QF and XW helped collection of data a; SB and FX contribute equally to the conception of this study and the final approval of the version to be published. All authors read and approved the final manuscript.

Received: 10 November 2009

Accepted: 27 February 2010 Published: 27 February 2010

\section{References}

1. Dosoretz DE, Katin MJ, Blitzer PH: Radiation therapy in the management of medically inoperable carcinoma of the lung: Results and implications for future treatment strategies. Int J Radiat Oncol Biol Phys 1992, 24:3-9.

2. Kamiyoshihara M, Hirai T, Kawashima O, Morishita Y: Resection of pulmonary metastases in six patients with disease-free interval greater than 10 years. Ann Thorac Surg 1998, 66:231-33.

3. Jeremic B, Classen J, Bamberg M: Radiotherapy alone in technically operable, medically inoperable, early-stage (I/II) non-small cell lung cancer. Int J Radiat Oncol Biol Phys 2002, 54:119-30.

4. Sibley G: Radiotherapy for patients with medically inoperable stage I non small cell lung carcinoma: Smaller doses and higher doses-A review. Cancer 1998, 82:433-38.

5. Sibley G, Jamieson T, Marks LB, Anscher MS, Prosnitz LR: Radiotherapy alone for medically inoperable stage I non-small-cell lung cancer: The Duke experience. Int J Radiat Oncol Biol Phys 1998, 40:149-54.

6. Mori Y, Kondziolka D, Flickinger JC, Kirkwood JM, Agarwala S, Lunsford LD: Stereotactic radiosurgery for cerebral metastatic melanoma: factors affecting local disease control and survival. Int J Radiat Oncol Biol Phys 1998, 42:581-89.

7. Morita K, Fuwa N, Suzuki Y, Nishio M, Sakai K, Tamaki Y, et al: Radical radiotherapy for medically inoperable non-small cell lung cancer in clinical stage I: A retrospective analysis of 149 patients. Radiother Oncol 1997, 42:31-6.

8. Cheung PC, Mackillop WJ, Dixon P, Brundage MD, Youssef YM, Zhou S: Involved-field radiotherapy alone for early-stage non-small-cell lung cancer. Int J Radiat Oncol Biol Phys 2000, 48:703-11.

9. Gauden S, Ramsay J, Tripcony L: The curative treatment by radiotherapy alone of stage I non-small cell carcinoma of the lung. Chest 1995, 108:1278-82. 
10. Slotman BJ, Njo KH, Karim AB: Curative radiotherapy for technically operable stage I nonsmall cell lung cancer. Int J Radiat Oncol Biol Phys 1994, 29:33-7.

11. Willner J, Baier K, Caragiani E, Tschammler A, Flentje M: Dose, volume, and tumor control prediction in primary radiotherapy of non-small-cell lung cancer. Int J Radiat Oncol Biol Phys 2002, 52:382-89.

12. Belderbos J, De Jaeger $K$, Heemsbergen W, Seppenwoolde $Y$, Baas $P$, Boersma $L$, et al: First results of a phase I/II dose escalation trial in nonsmall cell lung cancer using three-dimensional conformal radiotherapy. Radiother Oncol 2003, 66:119-26.

13. Uematsu M, Shioda A, Taira J, Wong J, Hama Y, Kusano S: Computed tomography (CT)-guided stereotactic radiation therapy (SRT) for Stage I non-small cell lung cancer (NSCLC): 8-year results of 50 initial patients. Int J Radiat Oncol Biol Phys 2003, 57:S281.

14. Onishi H, Araki T, Shirato H, Nagata Y, Hiraoka M, Gomi K, et al: Stereotactic hypofractionated high-dose irradiation for Stage I nonsmall cell lung carcinoma: Clinical outcomes in 245 subjects in a Japanese multiinstitutional study. Cancer 2004, 101:1623-31.

15. Zimmermann FB, Geinitz H, Schill S, Grosu A, Schratzenstaller U, Molls M et al: Stereotactic hypofractionated radiation therapy for Stage I nonsmall cell lung cancer. Lung Cancer 2005, 48:107-14.

16. Grills IS, Hugo G, Kestin LL, Galerani AP, Chao KK, Wloch J, et al: Imageguided radiotherapy via daily online cone-beam $C T$ substantially reduces margin requirements for stereotactic lung radiotherapy. Int $J$ Radiat Oncol Biol Phys 2008, 70(4):1045-56.

17. Wong JW, Sharpe MB, Jaffray DA, Kini VR, Robertson JM, Stromberg JS, et al: The use of active breathing control $(A B C)$ to reduce margin for breathing motion. Int J Radiat Oncol Biol Phys 1999, 44(4):911-9.

18. Xu F, Wang J, Bai S, Li Y, Shen Y, Zhong R, et al: Detection of intrafractional tumor position error in radiotherapy utilizing cone beam computed tomography. Radiother Oncol 2008, 89:311-9.

19. Van Herk M: Errors and margins in radiotherapy. Semin Radiat Oncol 2004, 14(1):52-64.

20. Sonke JJ, Rossil M, Wolthaus J, et al: Frameless stereotactic body radiotherapy for lung cancer using four-dimensional cone beam CT guidance. Int J Radiat Oncol Biol Phys 2009, 74:567-574.

21. Wulf J, Ulrich $H$, Ulrich O: Hypofractionated, high-doseradiation under stereotactic conditions in the stereotactic body frame: Accuracy of repositioning at 11 CT-simulations and 37 applications at the LINAC. Int J Radiat Oncol Biol Phys 1998, 42(Suppl 1):215

22. Negoro Y, Nagata Y, Aoki T, Mizowaki T, Araki N, Takayama K, et al: The effectiveness of an immobilization device in conformal radiotherapy for lung tumor: Reduction of respiratory tumor movement and evaluation of daily set-up accuracy. Int J Radiat Oncol Biol Phys 2001, 50:889-98.

23. Koshani R, Balter J, Hayman J, Henning G, van Herk M: Short-term and long term reproducibility of lung tumor position using active breathing control (ABC). Int J Radiation Oncology Biol Phys 2006, 65(5):1553-1559.

24. Uematsu M, Shioda A, Suda A, Tahara K, Kojima T, Hama Y, et al: Intrafractional tumor position stability during computed tomography (CT)-guided frameless stereotactic radiation therapy for lung or liver cancers with a fusion of CT and linear accelerator (FOCAL) unit. Int $J$ Radiat Oncol Biol Phys 2000, 48:443-8.

25. Guckenberger M, Meyer J, Wilbert J, Richter A, Baier K, Mueller G, et al: Intra-fractional uncertainties in cone-beam CT based image-guided radiotherapy (IGRT) of pulmonary tumors. Radiotherapy and Oncology 2007, 83:57-64.

26. Hanley J, Debois MM, Mah D, Mageras G, Raben A, Rosenzweig K, et al: Deep inspiration breath-hold technique for lung tumors: the potential value of target immobilization and reduced lung density in dose escalation. [J] Int J Radiat Oncol Biol Phys 1999, 45(3):603-11.

27. Sarrut D, Blodea V, Ayadi M, Badel JN, Ginestet C, Clippe S, et al: Nonrigid registration method to assess reproducibility of breath-holding with $A B C$ in lung cancer. [J] Int J Radiat Oncol Biol Phys 2005, 61(2):594-607.

28. Spoelstra F, De Koste JR, Vincent A, Cuijpers JP, Slotman BJ, Senan S: An evaluation of two internal surrogates for determining the threedimensional position of peripheral lung tumors. Int. J. Radiation Oncology Biol. Phys 2009, 74(2):623-629.

29. van der Geld Y, Lagerwaard F, van Sörnsen de Koste JR, Cuijpers JP, Slotman BJ, Senan S: Reproducibility of target volumes generated using uncoached 4-dimensional CT scans for peripheral lung cancer. Radiation Oncology 2006, 1:43, doi: 10.1186/1748-717X-1-43.
30. Wilson EM, Williams FJ, Lyn EB, Wong JW, Aird EG: Validation of active breathing control in patients with non-small cell lung cancer to be treated with CHARTWEL. Int J Radiat Oncol Biol Phys 2003, 57(3):864-84

31. Liu HH, Balter P, Tutt T, Choi B, Zhang J, Wang C, et al: Assessing respiration-induced tumor motion and internal target volume using four-dimensional computed tomography for radiotherapy of lung cancer. Int J Radiation Oncology Biol Phy 2007, 68(2):531-540.

32. Cheung PC, Sixel KE, Tirona R, Ung YC: Reproducibility of lung tumor position and reduction of lung mass within the planning target volume using active breathing control (ABC). Int J Radiat Oncol Biol Phys 2003, 57:1437-1442.

33. Panakis N, McNair HA, Christian JA, Ruheena M, Symonds TJ, Knowles C, et al: Defining the margins in the radical radiotherapy of non-small cell lung cancer (NSCLC) with active breathing control (ABC) and the effect on physical lung parameters. Radiotherapy and Oncology 2008, 87:65-73.

34. Wolthaus JW, Schneider C, Sonke J-J, Van Herk M, Belderbos JS, Rossi M, et al: Mid-ventilation CT scan construction from four-dimensional respiration-correlated $\mathrm{CT}$ scans for radiotherapy planning of lung cancer patients. Int J Radiat Oncol Biol Phys 2006, 65:1560-1571.

35. Uematsu M, Shioda A, Tahara K, Fukui T, Yamamoto F, Tsumatori G, et al: Focal, high dose, and fractionated modified stereotactic radiation therapy for lung carcinoma patients. Cancer 1998, 82:1062-70.

36. Arimoto T, Usubuchi H, Matsuzawa T, et al: Small volume multiple noncoplanar arc radiotherapy for tumors of the lung, head and neck and the abdominopelvic region. CAR'98 computer assisted radiology and surgery Tokyo; ElsevierLemke HU 1998, 257-61.

37. Nagata Y, Takayama K, Matsuo Y, Norihisa Y, Mizowaki T, Sakamoto T, et al: Clinical outcome of a phase I/II study of 48 Gy of stereotactic body radiotherapy in 4 fractions for primary lung cancer using a stereotactic body frame. Int J Radiat Oncol Biol Phys 2005, 63:1427-31.

38. Herfarth KK, Debus J, Lohr F: Stereotactic single dose radiation treatment of tumors in the lung. Radiology 2000, 217(Suppl):148.

39. Timmerman R, Papiez L, McGarry R, Likes L, DesRosiers C, Frost S, et al: Extracranial stereotactic radioablation: results of a phase I study in medically inoperable stage I non-small cell lung cancer. Chest 2003, 124(5):1946-55.

40. Fukumoto S, Shirato H, Shimzu S, Ogura S, Onimaru R, Kitamura K, et al: Small-Volume Image-Guided Radiotherapy Using Hypofractionated, Coplanar, and Noncoplanar Multiple Fields for Patients with Inoperable Stage I Nonsmall Cell Lung Carcinomas. Cancer 2000, 95:1546-53.

doi:10.1186/1748-717X-5-19

Cite this article as: Shen et al:: Hypofractionated radiotherapy for lung tumors with online cone beam CT guidance and active breathing control. Radiation Oncology 2010 5:19.

\section{Submit your next manuscript to BioMed Central and take full advantage of:}

- Convenient online submission

- Thorough peer review

- No space constraints or color figure charges

- Immediate publication on acceptance

- Inclusion in PubMed, CAS, Scopus and Google Scholar

- Research which is freely available for redistribution

Submit your manuscript at www.biomedcentral.com/submit
C Biomed Central 